\title{
Stopping the spread of measles
}

Despite the availability of a potent vaccine, outbreaks of measles continue to occur around the world. Two major factors contribute to this prevalence. First, the measles virus is highly transmissible and may be spread through the air in droplets produced by breathing, sneezing and coughing. Within 2 weeks of exposure to the virus, about $90 \%$ of unvaccinated individuals will develop infection. Second, vaccine coverage is incomplete because some people lack access while others decline vaccination. Once a person is infected with the virus, there is no effective treatment. For these reasons, the measles virus poses a concerning risk to public health. But epidemiologists working to eradicate the measles may now have a new tool at their fingertips. Researchers at Georgia State University and Emory University (both in Atlanta, GA), along with international collaborators, developed an antiviral drug that effectively protected ferrets from infection with canine distemper virus (CDV), a morbillivirus closely related to measles. The measles virus could not

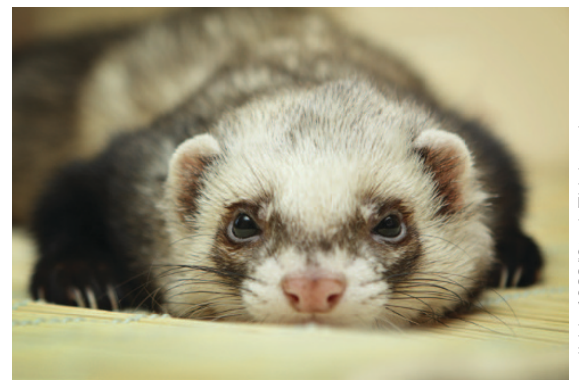

be tested in the ferret model because only primates are susceptible to it.

Ferrets were treated with the antiviral drug for 2 weeks beginning 3 days after exposure to CDV. The disease was completely suppressed, with all of the treated animals surviving the infection and developing immunity against future infection with CDV (Sci. Transl. Med. 6, 232ra52; 2014). The research team, led by Richard Plemper, next plans to test the drug's efficacy against the measles virus using squirrel monkeys.

The antiviral drug, called ERDRP0519, is intended not as a replacement for the measles vaccine but as a preemptive treatment for avoiding infection in unvaccinated individuals who may have been exposed to the virus. "We strongly support vaccination," Plemper told Scientific American, continuing, "This drug was not developed as an alternative to vaccination but rather as an additional weapon in our arsenal against the virus that may enable us to improve disease management and rapidly silence outbreaks." The researchers hope that ERDRP-0519 can be used in synergy with the measles vaccine to fill gaps in vaccine coverage. "Combined, they may one day succeed in eradicating measles totally," said Plemper in New Scientist.

One potential obstacle to achieving this goal is the possibility that strains of the virus may become resistant to ERDRP0519. Drug-resistant strains of measles virus in the lab were less transmissible or less virulent than the parent strain, however, leading the researchers to conclude that the clinical significance of such strains would be minimal.

Monica Harrington

\section{ENHANCING THE BRAIN'S RESTLIENCE AGATNST DEPRESSION}

A new study has uncovered a possible explanation for why some individuals are more likely than others to become depressed after experiencing stressful events. Researchers at Icahn School of Medicine at Mount Sinai (New York, NY) studied mice that had been subjected to 10 days of a 'chronic social defeat' paradigm, in which they are housed in cages with a dominant-aggressive rival. The response to this procedure differentiates mice that are susceptible to developing symptoms that mimic depression in humans from those that are resilient to developing these symptoms.

Ming-Hu Han and his team previously found that the difference between these mice depends on the activity of dopamine-producing neurons in a brain region called the ventral tegmental area (VTA). Hyperactivity of these neurons is linked to depressive symptoms such as avoidance of social contact, loss of motivation and lack of pleasure from rewarding activities. In a previous study, the team turned resilient rodents into susceptible ones, and vice versa, by stimulating or silencing their VTA dopamine neurons, respectively.

Underlying the neuronal hyperactivity in the susceptible mice is an increase in a particular excitatory current called $\mathrm{I}_{\mathrm{h}}$. Surprisingly, when the scientists measured the $I_{h}$ currents in the neurons of resilient mice, which fired normally, the currents were even larger. But the resilient mice also had stronger inhibitory currents to cancel out the excitatory ones; these well-balanced currents make the neurons resistant to perturbations. Han's team theorized that if they could boost the activity of hyperactive neurons even further, they could trigger a compensatory response to bring the firing of the neurons under control. "There's a saying in Chinese: If you push something to an extreme, the only way it can go is in the opposite direction," explained Han.

The researchers boosted the $I_{h}$ currents of the susceptible mice in three ways: by administering a drug, by genetically adding extra copies of a protein that drives these currents and by stimulating the activity of the neurons directly using optogenetics. With all three approaches, the VTA neurons started firing normally, and the depressive symptoms of the susceptible mice were reversed after 5 days (Science 344, 313-319; 2014).

Although this research enhances our knowledge about the mechanisms of resilience in the brain, Han cautions that an approach similar to the one used in this study could not be attempted for the treatment of depression in humans, because worsening depression symptoms could lead to an increased risk of suicide. 